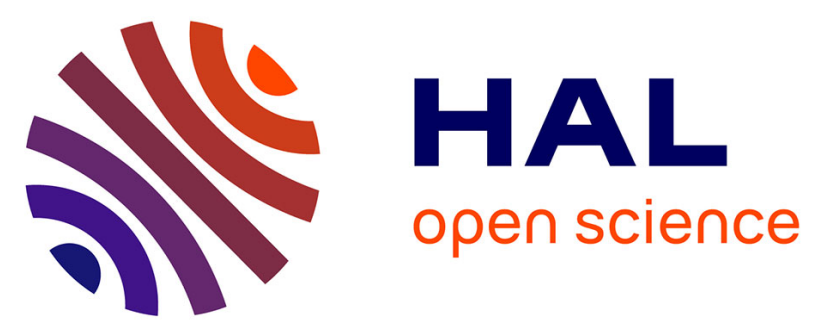

\title{
Design process of high dynamics multi-link flexible robot manipulators
}

\author{
Thomas Solatges, Mathieu Rognant, Sébastien Rubrecht, Eric Courteille, \\ Philippe Bidaud
}

\section{- To cite this version:}

Thomas Solatges, Mathieu Rognant, Sébastien Rubrecht, Eric Courteille, Philippe Bidaud. Design process of high dynamics multi-link flexible robot manipulators. ASME 2017 International Design Engineering Technical Conferences and Computers and Information in Engineering Conference, IDETC/CIE 2017, Aug 2017, Cleveland, OH, United States. 10.1115/DETC2017-67721 . hal01671672

HAL Id: hal-01671672

https://hal-univ-rennes1.archives-ouvertes.fr/hal-01671672

Submitted on 4 Mar 2019

HAL is a multi-disciplinary open access archive for the deposit and dissemination of scientific research documents, whether they are published or not. The documents may come from teaching and research institutions in France or abroad, or from public or private research centers.
L'archive ouverte pluridisciplinaire HAL, est destinée au dépôt et à la diffusion de documents scientifiques de niveau recherche, publiés ou non, émanant des établissements d'enseignement et de recherche français ou étrangers, des laboratoires publics ou privés. 


\section{DESIGN PROCESS OF HIGH DYNAMICS MULTI-LINK FLEXIBLE ROBOT MANIPULATORS}

\author{
Thomas Solatges \\ SITIA \\ Bouguenais 44340 \\ France \\ Email: thomas@solatges.fr
}

\author{
Mathieu Rognant \\ ONERA \\ Toulouse 31000 \\ France \\ Email: mathieu.rognant@onera.fr
}

\author{
Sébastien Rubrecht \\ SITIA \\ Bouguenais 44340 \\ France \\ Email: s.rubrecht@sitia.fr
}

\author{
Eric Courteille \\ INSA-LGCGM \\ RENNES 35043 \\ France \\ Email: eric.courteille@insa-rennes.fr
}

\author{
Philippe Bidaud \\ ONERA \\ Palaiseau 91123 \\ France \\ Email: philippe.bidaud@onera.fr
}

\begin{abstract}
This paper presents a design process based on an advanced flexible robots modeling tool associated with realistic actuators models and pre-defined control architecture. This process implements dedicated feasibility and performance indicators, which are used to evaluate a design and its sensitivity on the considered parameters. The proposed approach is illustrated with theoretical and experimental results obtained with the YAKA robot.
\end{abstract}

\section{NOMENCLATURE}

DOF Degrees Of Freedom.

EOA End Of Arm.

L\&R Launch and Recovery.

NDI Non-linear Dynamic Inversion.

UAV Unmanned Aerial Vehicle.

\section{INTRODUCTION}

This paper presents the design process used in the YAKA project to develop a highly dynamic robot manipulator. The aim of this project is to demonstrate the feasibility of Launching and
Recovering (L\&R) a small fixed-wing UAV from a naval vehicle with a robot manipulator. In this context the developed robot has to comply with ambitious specifications : a small footprint, an important workspace to compensate the ships movements, and high-speed on the end effector to minimize the relative velocity between the two vehicles.

In common industrial applications, robot manipulators are designed to be stiff enough to avoid flexibility issues, which implies massive links and joints, limiting therefore the reachable dynamics for high scale workspace applications. For this L\&R application, a classical rigid design leads to a dead end because such a structure would imply extremely massive links. This massive system would require unattainable actuator specifications, induce unreasonable costs, kinetic energy, mechanical interactions, energy consumption and safety questions.

To solve this issue, the retained design principle presented in this paper consists in reducing the moving mass of the robot, which implies using flexible links. However, to ensure the respect of the specifications, both mechanical constraints and control issue have to be considered together from the earliest design stages. 
Incoming technologies or specific approaches such as additive manufacturing of optimized structures [1] or cable-stayed structures [2,3] could offer interesting design opportunities. However, in an industrial industrial context, those technologies are not available. This article therefore focuses on designing structures made of regular section links and classic mechanical components. Material improvement and innovative structural design could still be associated with the proposed approach for specific demanding applications.

\section{Flexible robot design issues}

The design of flexible robots is a complex task as it requires to take into account aspects dramatically impacted by flexibility such as precision and stability, which are generally addressed separately in control synthesis phases. Regarding precision, static deflection produces a static error in the EOA position while the elasto-dynamic behavior produces vibrations that affect the EOA trajectory. Regarding stability, issues appear when flexibility is located in-between the actuators and the feedback sensors. Natural vibration modes excitation by the control law or external perturbations may also produce destructive resonance phenomena.

- Vibration modes: Flexible robots have natural vibration modes that reduce the structure longevity due to fatigue of materials and deteriorates the trajectory tracking performances. The proposed indicators are designed to take into account the vibration modes and evaluate their impact on the feasibility and performances of a design.

- Mechanical damping: The damping of flexible robots has a major influence on their mechanical behavior. Many active and passive damping techniques have been developed to increase the structural damping of robots [4] as it reduces the resonance phenomenon issues and offers better closed loop control robustness. However, those techniques require either extra actuators or specific developments on structural materials which increase the cost and complexity of the resulted system. Regarding the operational context, this work is restricted to the use of off-the-shelf available metal and composite components and does not consider adding extra vibration damping actuators.

- Mechanical resistance: Rigid robots are designed to be stiff enough regarding tip deflection and stability issues which in general lead to oversized structures regarding the mechanical resistance limit. When considering flexible robots, the mechanical resistance may be an important design constraint that must be considered during the design stage instead of a simple formality check on the final design.

- Mechanical specifications availability: In order to tackle the issues presented above, it is necessary to work with complex realistic elasto-dynamic models. The mass and stiffness parameters of construction material such as aluminum alloy or steel are usually well known. However, composite materials, ball bear- ings, gear boxes and other mechanical elements present much more complex behaviors. Moreover, the mechanical properties of such components are rarely completely and accurately described in product specifications. A sensitivity analysis on those uncertain parameters is performed in the proposed design process in order to anticipate the performances variations due to modeling inaccuracies.

In order to address the issues presented above, this paper presents a design process based on advanced flexible robots modeling tools [5] with realistic actuators and pre-defined control architecture. This process implements dedicated feasibility and performance indicators, which are used to evaluate a design and its sensitivity on the considered parameters. We paid attention to define indicators that are physically accurate and relevant to the application. The suggested indicators and the full system based evaluation process does extend rigid robots classical design methods $[6,7]$.

After a presentation of the case study, the system modeling is detailed. The design approach proposes an iterative process making use of this model, which performances are evaluated and discussed in the design analysis section. The proposed approach is then illustrated with theoretical and experimental results obtained with the YAKA robot.

\section{CASE STUDY: THE YAKA ROBOT}

The proposed approach intervenes in the YAKA ${ }^{1}$ project, a large-scale Launch and Recovery (L\&R) robot arm project held by SITIA ${ }^{2}$ company.

The goal of the YAKA project is to develop a demonstrator robot manipulator able to launch and recover a $5 \mathrm{Kg}$ fixedwing UAV from a naval ship. The unusual performance requirements for this application motivates the proposed advanced design approach. FIGURE 1 shows the considered recovery scenario where an UAV is reaching a ship with a differential speed of up to $15 \mathrm{~m} / \mathrm{s}$. The YAKA robot generates a trajectory to catch the UAV and then dissipates its kinetic energy while compensating deck movements and preserving the UAV integrity. Industrial and practical constraints associated with the project are also considered in the proposed contribution.

The YAKA robot, developed for the L\&R application, reaches trajectories with an EOA speed of 10 to 14 meters per second in a wide workspace. It is about 7.5 meters long and weighs less than $150 \mathrm{Kg}$ while fast moving parts are lighter than $20 \mathrm{Kg}$. Consequently it presents unusually low frequency vibration modes and high dynamic capabilities which impose specific design and control techniques. The YAKA robot design has been a challenging iterative process that required innovative developments. The proposed approach is based on this experience, it is

\footnotetext{
${ }^{1}$ www.sitia.fr/yaka

${ }^{2}$ Robotics and test rig company
} 


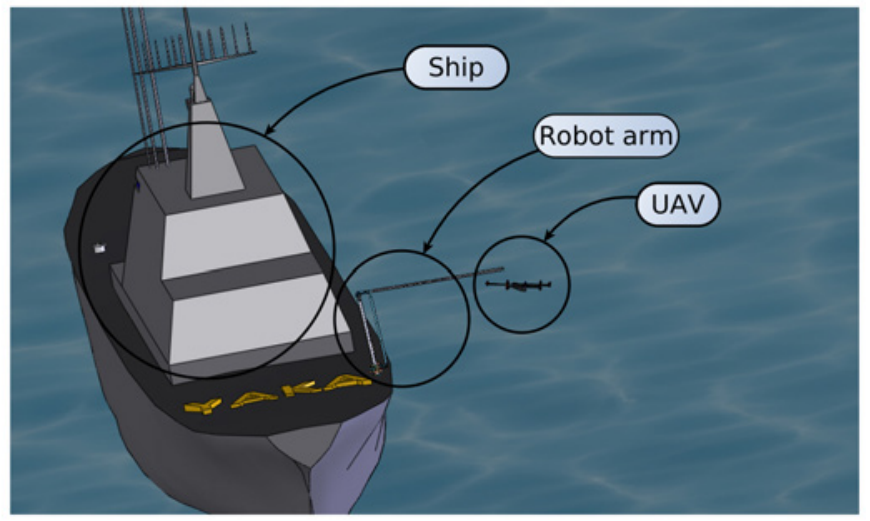

FIGURE 1. YAKA PROJECT - RECOVERY OPERATION

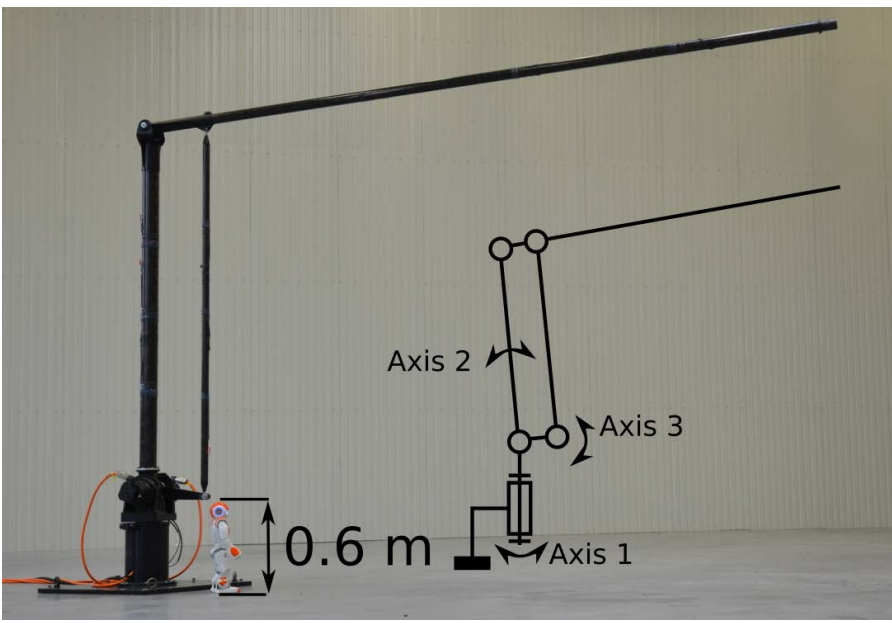

FIGURE 2. YAKA ROBOT NEXT TO NAO

illustrated with theoretical and experimental results.

FIGURE 2 shows the YAKA robot next to a $60 \mathrm{~cm}$ tall Aldebaran Nao robot. The main features of the robot are given below:

- 3 joints actuated by brushless DC motors and gearbox transmissions

- $15 \mathrm{~m} / \mathrm{s}$ maximum EOA speed

- Total mass $<150 \mathrm{Kg}$, moving parts $<20 \mathrm{Kg}$ moving parts

- Closed loop kinematics

- 4x2x2m UAV recovery window (workspace area in which the maximum speed can be reached)

- $7.5 \mathrm{~m}$ total length

\section{SYSTEM MODELING}

The proposed approach is based on advanced tools to model the complete robotic system. It takes into account the flexible

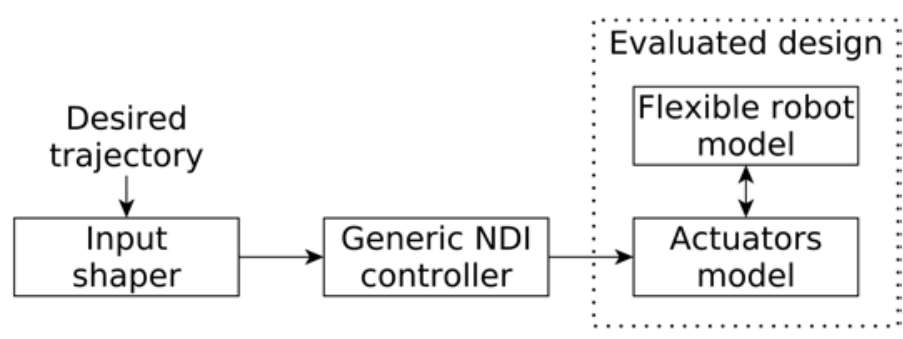

FIGURE 3. SYSTEM MODEL

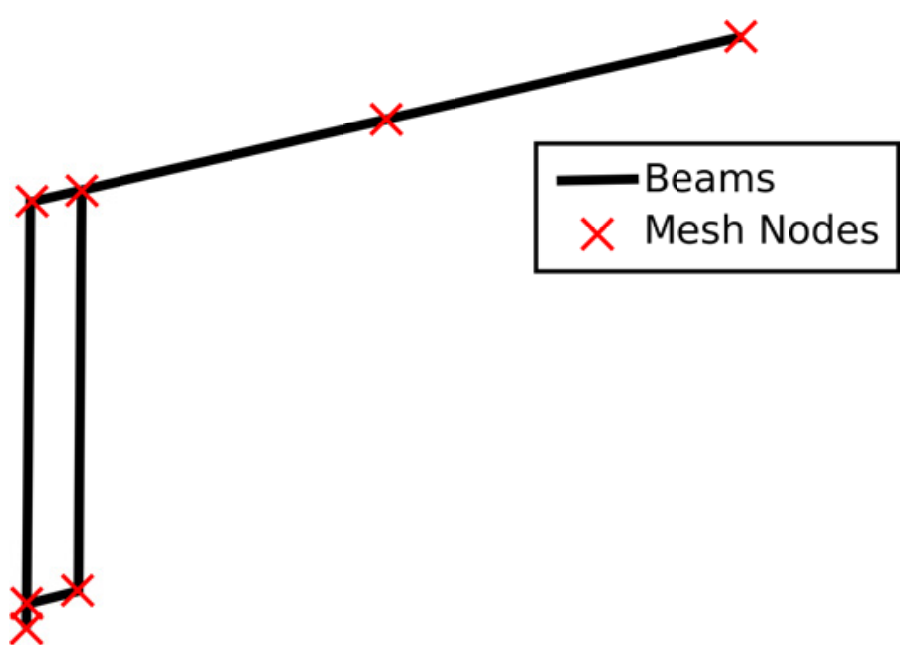

FIGURE 4. YAKA ROBOT MESH

structure dynamics, actuators and controller. FIGURE 3 summarizes the different system elements described in the following section.

\section{Input parameters}

The symbolic model relies on parameters describing the mechanical characteristics of the flexible system. The Khalil and Kleinfinger [8] table and the mechanical parameters respectively contain the kinematics and mechanical description of the robot.

The flexible links are divided in a mesh of Euler-Bernoulli beams, each defined by the parameters:

- Length $L$

- Cross-sectional $S$

- Quadratic moments $I_{x}, I_{y}$, and $I_{z}$

- Young's modulus $E$

- Coulomb's modulus $G$

- Density $\rho$

- Localized mass at each nodes $M_{1}$ and $M_{2}$ (if relevant)

- Structural Rayleigh damping coefficients $\alpha$ and $\beta$ 
FIGURE 4 shows the chosen mesh for modeling the YAKA robot.

The passive and active flexible joints are described by:

- Rotational stiffness $\mathrm{Krx}, \mathrm{Kry}, \mathrm{Krz}$ and damping $\mathrm{Cr} x, \mathrm{Cry}, \mathrm{Crz}$

- Axial translational stiffness $K a$ and damping $C a$

- Radial translational stiffness $\mathrm{Kr}$ and damping $\mathrm{Cr}$

\section{Obtained mechanical symbolic model}

The full dynamic model is obtained given the parameters presented above. The obtained model is given as the differential equation (1), according to [5]:

$$
\begin{array}{r}
\left(\begin{array}{cc}
\mathbf{M}_{q q} & \mathbf{M}_{q u} \\
\mathbf{M}_{q u}^{T} & \mathbf{M}_{u u}
\end{array}\right)\left(\begin{array}{c}
\ddot{\mathbf{q}} \\
\ddot{\mathbf{u}}
\end{array}\right)+\left(\begin{array}{cc}
\mathbf{0} & \mathbf{0} \\
\mathbf{0} & \mathbf{C}_{u u}
\end{array}\right)\left(\begin{array}{l}
\dot{\mathbf{q}} \\
\dot{\mathbf{u}}
\end{array}\right)+\left(\begin{array}{cc}
\mathbf{0} & \mathbf{0} \\
\mathbf{0} & \mathbf{K}_{u u}
\end{array}\right)\left(\begin{array}{l}
\mathbf{q} \\
\mathbf{u}
\end{array}\right) \\
=\left(\begin{array}{c}
\Gamma \\
\mathbf{F e x t}
\end{array}\right)+\left(\begin{array}{l}
\mathbf{F c}_{q} \\
\mathbf{F c}_{u}
\end{array}\right)+\left(\begin{array}{c}
\mathbf{F} \mathbf{g}_{q} \\
\mathbf{F g}_{u}
\end{array}\right)
\end{array}
$$

With:

- $\mathbf{q}$ the vector of joints positions

- $\mathbf{u}$ the vector of elastic nodal displacements

- $\mathbf{M}_{i j}$ the distribution of mass on the rigid DOF, elastic DOF and coupling between rigid and elastic DOF (depends on $\mathbf{q}$ )

- $\mathbf{K}_{u u}$ the stiffness matrix (depends on $\mathbf{q}$ )

- $\mathbf{C}_{u u}$ the structural and joints damping (depends on q)

- $\Gamma$ the actuators torque

- Fext the external forces applied at each node of the mesh

- $\mathbf{F}_{c}$ the centrifugal and Coriolis effect (depends on $\mathbf{q}$ and $\dot{\mathbf{q}}$ )

- $\mathbf{F}_{g}$ the gravity forces (depends on $\mathbf{q}$ )

It describes the elasto-dynamic behavior of the mechanical structure taken apart. The actuators and controller models are proposed below.

\section{Actuators and transmission model}

The actuators are complex components that usually include an electric motor that produces a low torque at high rotation rate and a reducer that adapts this mechanical power to the required links dynamics. If considering other types of actuators and transmission, one could refer to [9] for various actuators models.

At the design stage, the mechanical parameters of complex actuators models are not known neither specified in supplier data sheets. The proposed model takes into account the Input inertia $I_{\text {in }}$, output inertia $I_{\text {out }}$, reduction ratio $R$, input viscous damping $F v_{\text {in }}$ and output viscous damping $F v_{\text {out }}$.

The axial stiffness and damping are respectively given by the joints parameters $\mathrm{Krz}$ and $\mathrm{Ca}$ taken from the mechanical model.

The obtained actuator model is associated with the mechanical model to characterize the coupled behavior of the actuators and the mechanical structure.

\section{Chosen control architecture}

The considered system uses a generic control architecture based on a joint controller and a command shaper:

- A Non-linear Dynamic inversion of the rigid robot model [8] ensures closed loop performances of the system approximated to rigid dynamics. This controller operates at the joint level on the motor side, which avoids stability issues encountered when controlling the EOA position in presence of flexibility located inside the control loop. The desired dynamic response time and overshoot are imposed by the feedback gains given the application specifications. This controller only depends on the application and not on the design as the closed loop system behaves as a double integrator, regardless the properties of the mechanical system. Thereby it can be used during the design process for comparative performances evaluation.

- An input shaper [10] is implemented to reduce the vibrations of the flexible structure. The input shaper parameters are determined given the natural frequencies and damping of the structure computed from the mechanical numeric model specific to each design. This method reduces the residual vibrations of flexible DOF induced by the joint trajectory of rigid DOF. A predicted modeling error can be considered to anticipate the real performances obtained with this method.

This control architecture is interesting as it can be automatically implemented and tuned, which allows for evaluating a batch of designs with a similar controller behavior. Moreover, rigid NDI [8] and Input Shaping techniques [10] has proven interesting performances in practical cases for respectively rigid and flexible systems. This choice ensures that minimal performances can be reached with the real robot.

Control design of flexible robot manipulators have received many theoretical efforts $[11,12]$ that could be implemented on the real robot to increase the closed loop performances. However, those techniques usually rely on extra sensors, heavier control schemes and complex non-linear observers, which is rarely compatible with short-term developments and/or cost effective industrial projects.

\section{Simulation model}

The full-scale model described above does not allow performing time domain simulation because of the size of the considered state vector and induces high frequency modes.

Considering the required control bandwidth $\omega_{c}$, all modes appearing at a frequency higher than three times $\omega_{c}$ can be neglected $[11,13]$. Modal truncation and an interpolation process inspired by [14] provides an approximated modal basis $\mathbf{P}(\mathbf{q})$ over the workspace of the robot. The reduced model (2) is then obtained and used for simulation: 


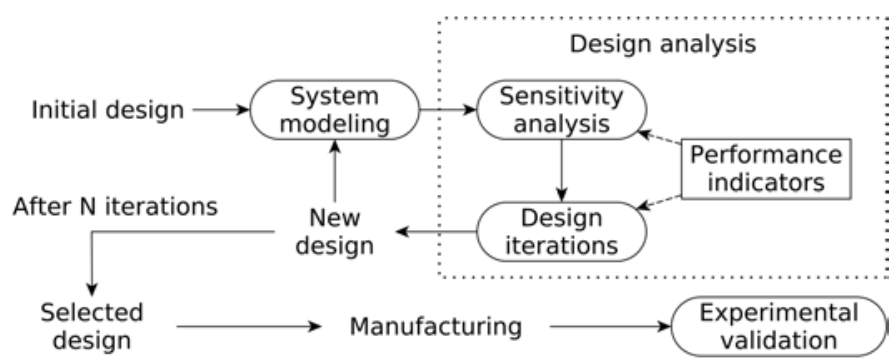

FIGURE 5. DESIGN PROCESS

$$
\begin{aligned}
& \left(\begin{array}{c}
\Gamma \\
\mathbf{P}^{T} \mathbf{F e x t}
\end{array}\right)+\left(\begin{array}{c}
\mathbf{F c}_{q} \\
\mathbf{P}^{T} \mathbf{F c}_{u}
\end{array}\right)+\left(\begin{array}{c}
\mathbf{F g}_{q} \\
\mathbf{P}^{T} \mathbf{F} \mathbf{g}_{u}
\end{array}\right)= \\
& \left(\begin{array}{cc}
\mathbf{M}_{q q} & \mathbf{M}_{q u} \mathbf{P} \\
\mathbf{P}^{T} \mathbf{M}_{q u}^{T} & \mathbf{P}^{T} \mathbf{M}_{u u} \mathbf{P}
\end{array}\right)\left(\begin{array}{c}
\ddot{\mathbf{q}} \\
\ddot{\eta}
\end{array}\right)
\end{aligned}
$$

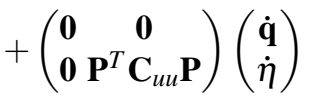

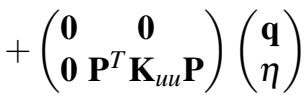

The modal variable $\eta=\mathbf{P}^{T} \mathbf{u}$ is the new flexible state vector that represents the contribution of each mode. Equivalent diagonal mass and stiffness matrices can be defined as:

$$
\begin{aligned}
\mathbf{M}_{\mathbf{e q}} & =\mathbf{P}^{T} \mathbf{M}_{u u} \mathbf{P} \\
\mathbf{K}_{\mathbf{e q}} & =\mathbf{P}^{T} \mathbf{K}_{u u} \mathbf{P}
\end{aligned}
$$

A simulation model is created from the reduced model (2) and allows for performing simulations, taking as input the reference trajectory and external forces applied at any node of the mesh. The time domain simulation computes the evolution of the rigid movements, elastic displacements, actuators torque, actuators speed and control variables.

In the YAKA application context, it is possible to simulate typical launch and recovery trajectories considering the interaction forces with the UAV.

\section{DESIGN PROCESS}

The proposed design process presented on FIGURE 5 starts from a preliminary robot design and leads to an improved design. The design objectives and process steps are detailed in the following sections.

\section{Practical context}

The design process is presented in an industrial context with specific constraints and objectives:

- The exploration space is limited to the available commercial components and classic manufactured mechanical parts. The joints and actuators are selected in a database of available components such as motors, transmissions, bearings. The links are constant profile beams such as tubes, cylinders, rods... The different parts are made of common materials such as metals, aluminum alloys and composites.

- The control architecture is based on state-of-the-art algorithms compatible with limited sensors and computational resources.

- The design objectives are specific to each project and may consider focus on various points such as unit cost for mass production, delays for short-term projects, finding a feasible solution for challenging research project or reaching an optimal solution. Each particular case reduces the considered solutions to a limited number of design options.

\section{Considered system}

While classic design techniques study the mechanical structure, the actuators and the controller separately, the proposed approach deals with the complete system. Considering the realistic behavior of the complete closed loop system allows for taking into account the flexible robots design issues.

FIGURE 3 shows the different elements of the considered system.

\section{Application specifications - process input}

The considered application specifications induce performance requirements of the system such as:

- The geometric workspace volume

- The maximum operational speed and acceleration in specified workspace areas

- Specific operational trajectories with associated end of arm trajectory tracking precision

- Natural insensitivity to a given external solicitation (no natural mode frequency close to external vibration sources frequency)

\section{Initial mechanical design - process input}

The design process takes as input an initial feasible design with respect to specifications without taking into account the system flexibility. This preliminary step is not detailed in this contribution, it can be achieved following classic mechanics and rigid robots design rules $[6,7]$. One may also use advanced rigid design optimization techniques such as [15-17] to perform this step. 
This initial design takes into account the specified geometric workspace, dexterity indices and acceleration radius [7] which satisfies the application requirements while considering the system infinitely stiff. The evaluation of those criteria gives a range of admissible values for the mechanical parameters of the robot.

The initial design should be feasible regarding project constraints, it provides a starting point with:

- The chosen kinematics parameters and allowed variation range. For instance, each link is given with a nominal length and extreme admissible values.

- Actuators with nominal specifications and possible alternatives

- Links nominal sections, materials, dimensions and possible alternatives.

This preliminary design induces constraints on the links lengths given the application specifications. However, links sections and material properties can be chosen from a large set of standard materials and profiles. The design steps presented below explores in between the given boundaries in order to evaluate and improve the design considering the behavior of the complete flexible system with realistic actuators and controller.

\section{Design steps}

FIGURE 5 presents the proposed design process divided in different steps. The initial design is first modeled following the proposed modeling approach. The obtained model is then evaluated with respect to performance indicators. A sensitivity analysis of the parameters on the design performances makes recommendations to improve the design. The iterations of this process are performed until reaching the desired performances or a local optimum.

\section{Obtained design - process output}

The final design obtained after the iterative process comes with quantified performances given by the performance indicators.

\section{DESIGN ANALYSIS}

Given the full system model and simulation tool, the goal of this design step is to evaluate a design with respect to quantifiable performance and feasibility indicators. A sensitivity analysis of the input parameters on those indicators proposes an improved version of the design.

\section{Sensitivity analysis}

A sensitivity analysis is pursued in order to reach 2 design objectives:

- Improve the considered design: evaluate the sensitivity of the tunable parameters on the performance and feasibility indi- cators gives the opportunity to improve the design by changing those parameters. The link lengths, cross-sectional, material and joint stiffness are in general the parameters that are the most sensitive on the system performances.

- Evaluate the impact of unknown or uncertain parameters on the overall design performances in order to minimize the performance variations between the selected design (based on suppliers specifications) and the real manufactured robot. Structural damping of composite materials, damping in the passive and actuated joints, stiffness of different commercial components such as bearings are most often not given in the products specifications or given with a low accuracy. Targeting those uncertain parameters which have an important influence on the design is necessary to evaluate the design process reliability and anticipate unavoidable variations between the design model and the real robot. Choosing well-defined components such as spindle bearings instead of classic bearing and high quality actuators with complete data sheets is a way to reduce the design uncertainties. Another solution is to purchase samples of unknown components and perform identification tests to determine its mechanical properties, but it may be prohibitive for delays and costs reason.

\section{Feasibility indicators}

The design evaluation mostly focuses on the performance of the design, but a first study is performed on feasibility indicators. Evaluating the feasibility of a design with respect to technological and project constraints assures that the design steps lead to an acceptable solution.

- The maximal stress in each link and joint loads are computed from the system model considering a worst case loading scenario: maximal external forces with maximal acceleration and maximum robot payload. The resulting maximal mechanical stress is compared with maximal admissible stress with a safety factor.

- A classic finite element analysis software is used in a second phase to validate the mechanical resistance of mechanical parts and interfaces given the computed mechanical loads.

- The actuators contribution performance indicators presented below are also evaluated as feasibility indicators by checking the compatibility with available actuator specifications.

- All the feasibility aspects of a design cannot be formalized into indicators, but the classic considerations of mechanical engineering and project management are considered during the design process.

\section{Model based performance indicators}

The mechanical model of the flexible structure (1) provides general information about the system. A first evaluation is made on the isolated flexible structure without considering the actuators and controller behavior.

- Vibration modes commandability: 


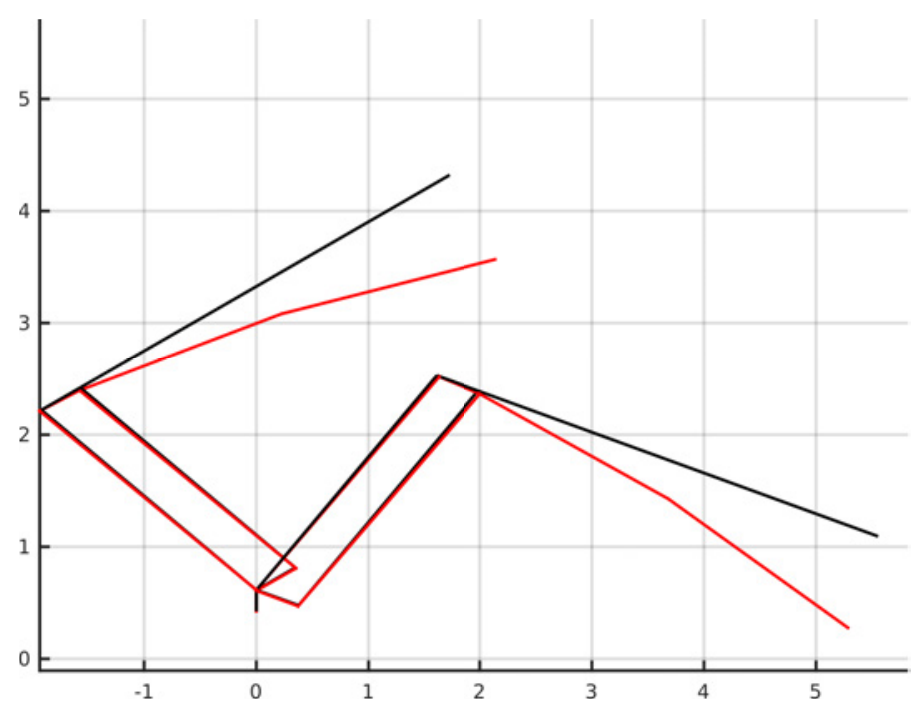

FIGURE 6. MODE SHAPE EVOLUTION

Evaluating the eigenvalues and eigenvectors of $\mathbf{M}_{u u}^{-1} \mathbf{K}_{u u}$ gives the modes frequency and modes shapes of the flexible structure. The frequency of the first vibration mode is often considered when the design objective is to avoid elasto-dynamic issues by placing the first mode frequency higher enough than the control bandwidth to be negligible. That way the robot could be considered rigid $[11,12]$.

The modes shapes given by the eigenvectors represent the effect of each vibration mode on the EOA position and also about the coupling between natural modes and actuated joints which give information about the system commandability. FIGURE 6 shows the second mode shape evolution of the YAKA robot over the workspace. It is represented in 2 dimensions as it is invariant by rotation around the first actuated joint (AXIS 1 shown FIGURE 2).

- Environment vibration compatibility: The natural frequencies of complex systems are evaluated considering the compatibility with the robot environment. In many practical cases, the environment of the robot produces vibrations that must be taken into account to avoid harmful resonance phenomena. On specific applications, a coupled modal analysis is needed to ensure the compatibility of the system with its environment.

For instance, our case study considers the robotic system on a ship deck with its own natural frequencies and vibratory signature generated by the propulsion system.

- Input shaping residual vibrations: The chosen control architecture presents a known robustness to modeling errors [10]. Evaluating the relative evolution of modes frequencies on the workspace allows to compute the maximal residual vibration level induced by the robot own movements (without considering external perturbations). FIGURE 7 shows the evolution of one
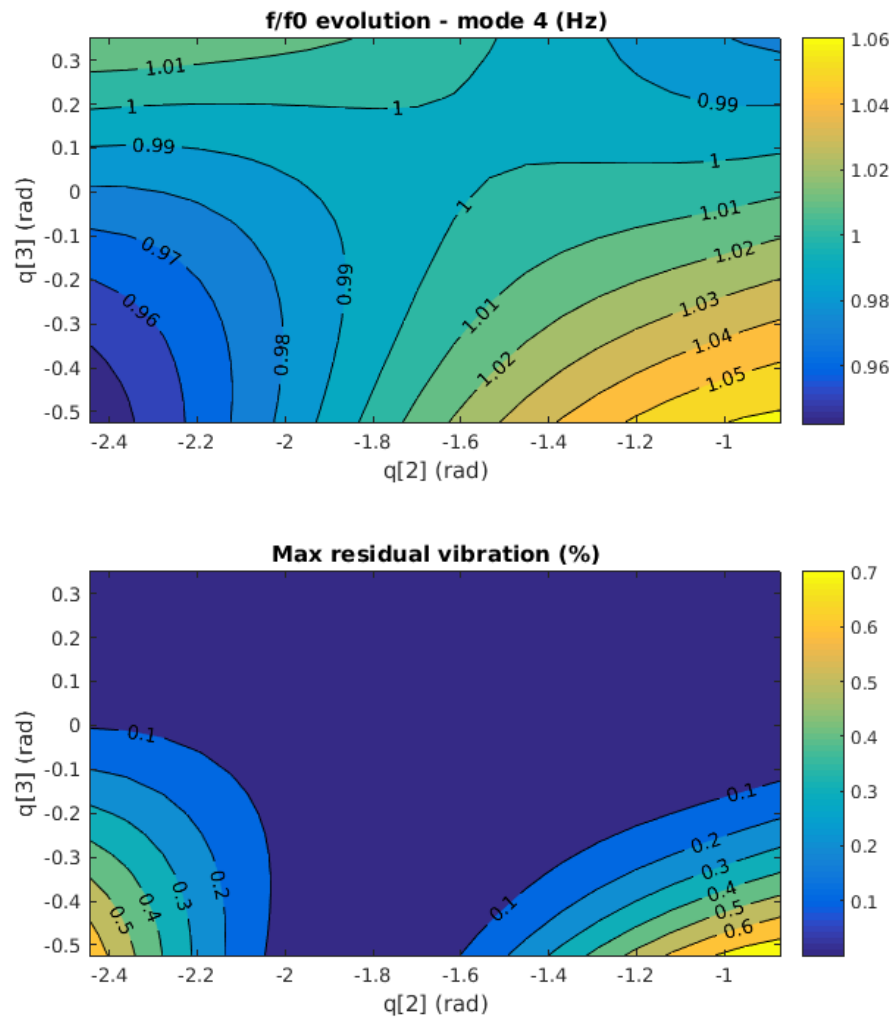

FIGURE 7. MODE FREQUENCY EVOLUTION AND INDUCED MAXIMUM RESIDUAL VIBRATION

natural mode frequency of the YAKA robot over the considered workspace and the associated maximal residual vibrations.

\section{Simulation based performance indicators}

The simulation model (2) is exploited on realistic scenarios with desired trajectories and external perturbations defined from application specifications. Performance indicators are computed to evaluate the design on a realistic scenario with an accurate model that considers the full closed loop system behavior.

The presented indicators are illustrated with simulation results of the YAKA robot performing a typical launch trajectory that involves all the robot axes in a highly dynamic and high speeds movement on FIGURES 8,11,12.

- Actuators power consumption: The actuators power and energy consumption for a given task is computed from the instantaneous absorbed power and its integral over time. The maximum instantaneous power and root mean square power consumption of actuators are in general limited by thermal considerations. The total energy needed to achieve the task is interesting for power efficiency considerations. Those indicators are also useful for specifying the power supply system.

- Modal energy: Considering the flexibility of the system, 
one important concern is the energy involved in vibrations as it influences the stability and the reliability of the system. The modal energy [18] is computed as the integral of the instantaneous kinetic and potential energy held in the flexible degrees of freedom. It is compared to the total energy consumption presented above to evaluate the energetic distribution between rigid and flexible dynamics. This indicator should in general be minimized to reduce vibrations, improve robustness and power efficiency.

$$
\begin{gathered}
E c=\frac{1}{2} \dot{\eta}^{T} \text { Meq } \dot{\eta} \\
E p=\frac{1}{2} \eta^{T} \mathbf{K e q} \eta \\
\text { Modal energy }=\int E c(t)+E p(t) \mathrm{d} t
\end{gathered}
$$

- EOA trajectory tracking error: The maximum trajectory tracking error of the robot during a task is another concern that is frequently specified in robotic applications. FIGURE 8 shows the EOA desired trajectory, real trajectory on each Cartesian direction and the total error (amplified 10 times) for a given YAKA launch trajectory. This result is obtained assuming a pessimistic natural frequency identification error of $20 \%$ considered for the input shaper tuning [10], showing its effects on the EOA trajectory tracking error. A perfect identification of the system natural modes completely cancels out residual vibrations (after the trajectory), and minimizes the EOA trajectory tracking errors.

\section{Design iterations}

The sensitivity analysis of the parameters on the performance indicators give a score for the actual design and possible improvement opportunities. A new design is then obtained thanks to those results while taking into account feasibility and project constraints such as availability, costs and manufacturing feasibility.

A new design is proposed and the process can be iterated until obtaining satisfactory performances or a local optimum.

Depending on the specifications and design constraints, one could identify that the problem is unsolvable starting from the given initial design. Then, either specifications, initial design and/or technological constraints should be reconsidered.

\section{EXPERIMENTAL RESULTS}

The different tools presented in this paper have been used for the real case study of the YAKA project. This section proposes experimental results obtained in this context.

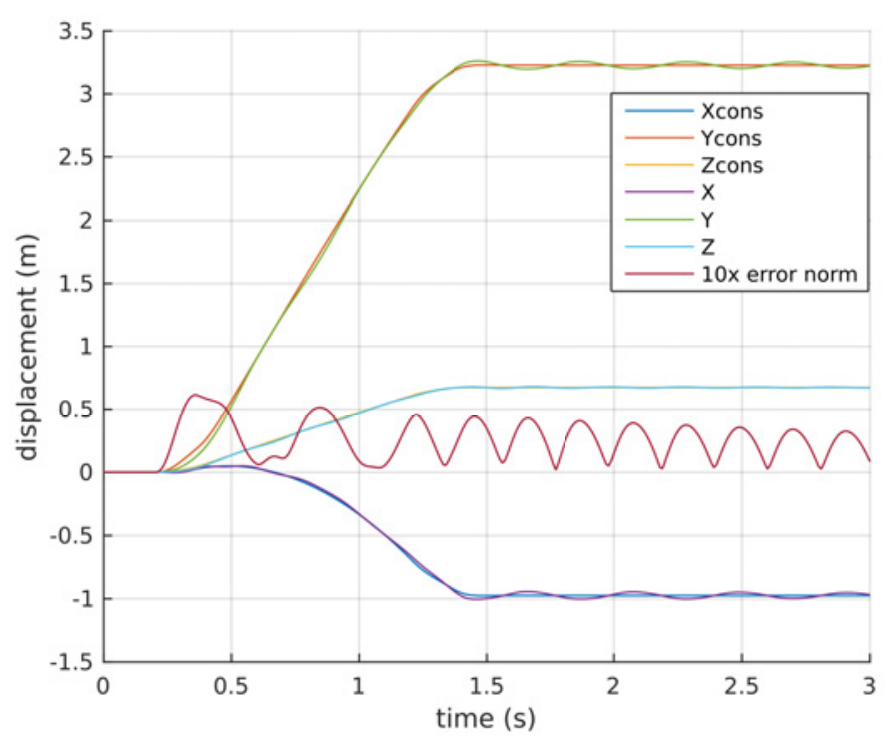

FIGURE 8. EOA TRAJECTORY TRACKING ERROR

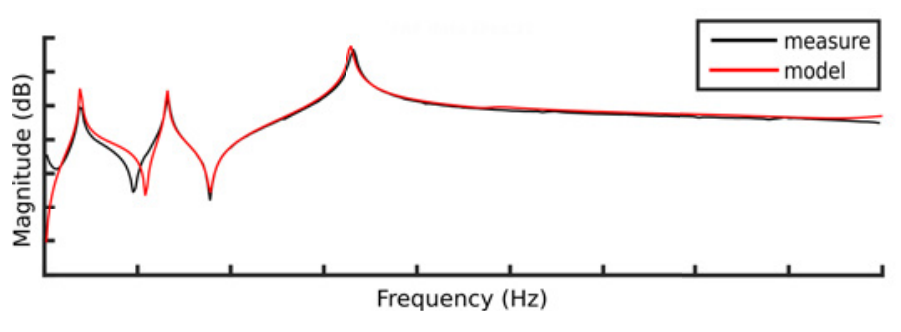

FIGURE 9. MODEL VALIDATION

\section{Model validation}

An experimental modal analysis allowed identification of the various mechanical parameters of the YAKA robot by correlating the model with the measures. A frequency response function of the model compared to the measured one is presented FIGURE 9. The obtained results are satisfactory, which validates the choice of the robot description and the accuracy of the symbolic modeling tool [5].

\section{Control architecture validation}

The proposed control architecture using NDI and input shaping techniques have been implemented in the real robot controller and the performances have been evaluated by measuring the EOA acceleration norm on a typical trajectory with an inertial measurement unit. FIGURE 10 shows experimental results that illustrate the interest of using an input shaper to reduce the structure vibrations. On this highly dynamic trajectory, the EOA acceleration is reduced by around $200 \%$ during the trajectory (0s 


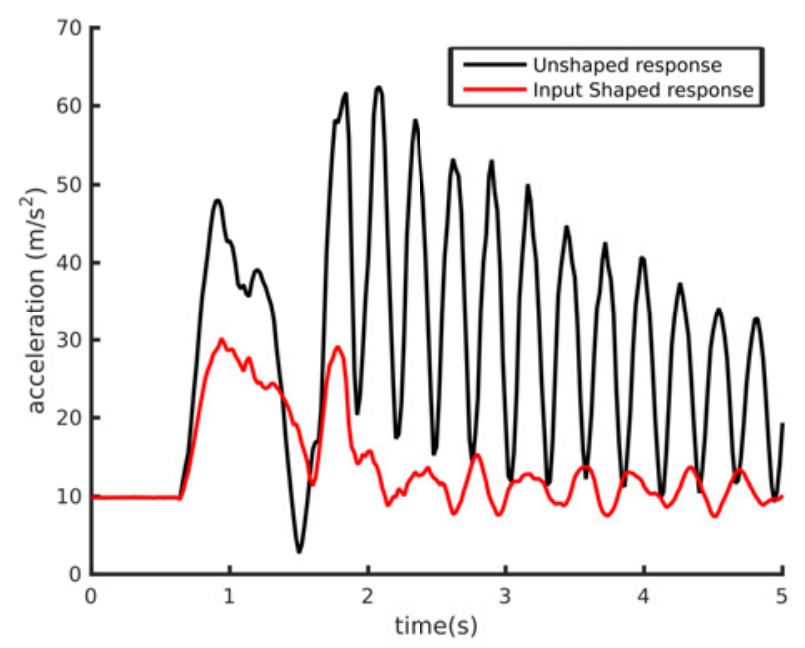

FIGURE 10. EOA ACCELERATION ON A TYPICAL TRAJECTORY

- 2s) and residual vibrations after the end of the movement (2s end) are reduced by more than $1000 \%$.

\section{YAKA application results}

The proposed design approach has been applied in a practical case and helped taking up the challenge of launching and catching an UAV with a robot arm. FIGURE 13 shows a picture of a successful recovery operation taken during outdoor validation tests.

\section{Comparative results}

In this section, the proposed performance indicators are computed for four different designs:

- The initial YAKA robot design made of aluminum links with components properties provided in the product specifications, unknown joints are considered rigid.

- The initial YAKA robot design made of aluminum links with mechanical joints properties identified on the real YAKA robot.

- The chosen YAKA robot design made of carbon links with components properties provided in the product specifications, unknown joints are considered rigid.

- The real YAKA robot model with completely identified mechanical parameters.

FIGURE 11 shows power indicators normalized to the maximal value. It illustrates the interest of using lightweight carbon links instead of aluminum links as it reduces both actuators RMS power and total energy. The sensitivity to uncertain parameters is illustrated by the difference between the results considering specified or identified parameters. The chosen lightweight car-

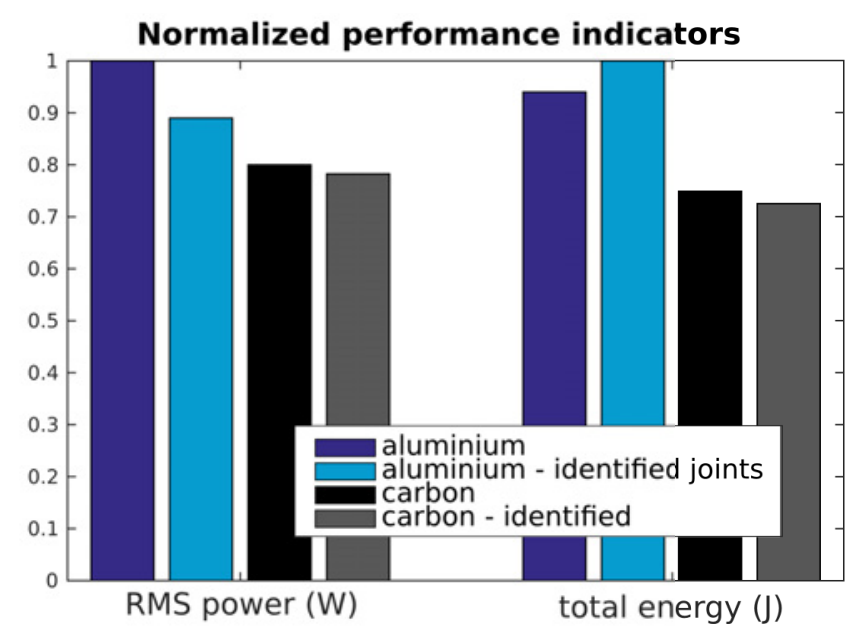

FIGURE 11. COMPARATIVE ENERGETIC INDICATORS

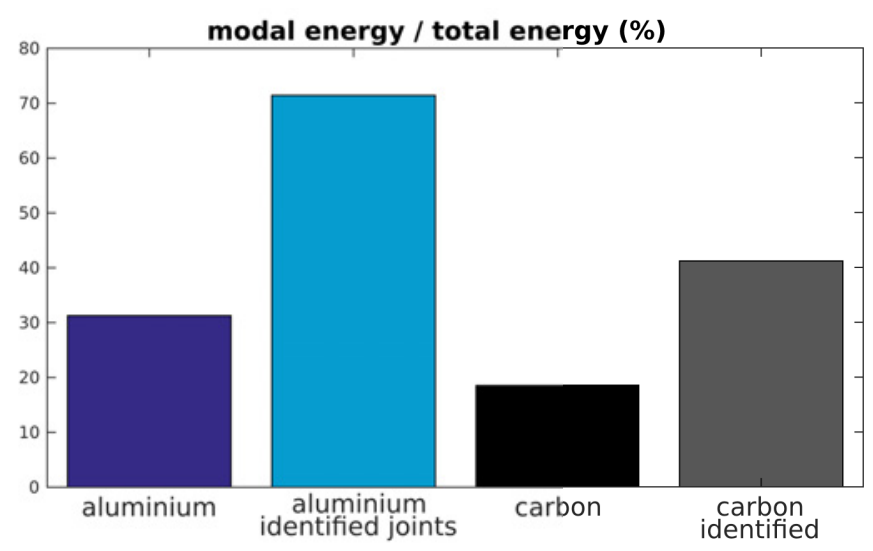

FIGURE 12. COMPARATIVE MODAL INDICATOR

bon links design reduces the global inertia coupled with the flexible joints and therefore reduces the sensitivity to uncertain joint mechanical properties.

FIGURE 12 shows the relative amount of modal energy to the total actuators energy. The results show that this indicator is very sensitive to joint uncertain parameters. Considering the identified stiffness parameters instead of taking rigid joints assumption reduces the global stiffness of the robot and therefore increases the modal energetic participation.

\section{CONCLUSION}

The proposed design process has given interesting results on the presented case study and allowed to successfully design the YAKA robot, despite its ambitious specifications.

The proposed indicators have shown their ability to assess 


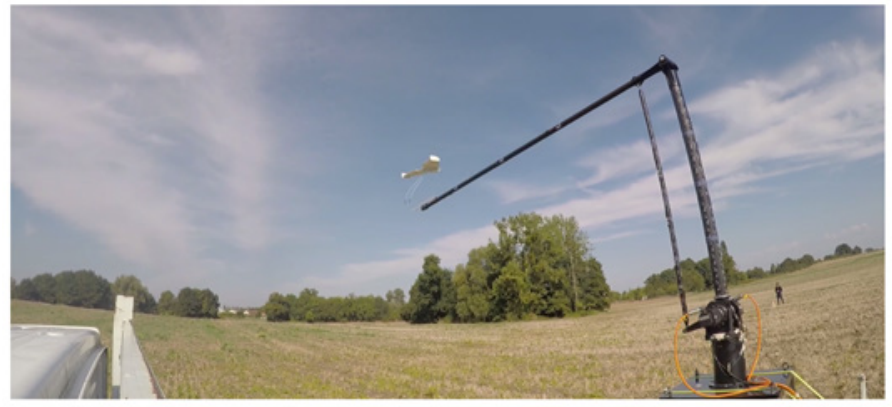

FIGURE 13. YAKA APPLICATION

the performance of a flexible robot design according to a given application. The sensitivity of these indicators allows for anticipating in the earliest design steps the performance variations implied by the lack of available information and the uncertainty on the mechanical properties.

The chosen control architecture, based on a non-linear dynamic inversion of the rigid model and input shaping techniques, provided an interesting reference controller for evaluating the reachable performances by the closed loop system.

Experimental data from the YAKA robot confirmed the relevance of the used modeling tools and the implemented control architecture.

This work was carried out in an industrial context, which motivated the use of standard mechanical components and control laws using few sensors and actuators. Future work could focus on extending this design process by including complex mechanical components, such as piezoelectric actuators or sensors, and their associated active damping control laws.

\section{ACKNOWLEDGMENT}

This work is partly supported by the French Ministere $d u$ Redressement Productif through the DGA RAPID Project L\&R YAKA, grant 132906095.

\section{REFERENCES}

[1] Laurent CHOUGRANI, Philippe VERON, Jean-Philippe PERNOT, S. A., 2016. "Parts internal structure definition using lattice patterns optimization for mass reduction in additive manufacturing". In Tools and Methods for Competitive Engineering.

[2] Pott, A., Mütherich, H., Kraus, W., Schmidt, V., Miermeister, P., and Verl, A., 2013. "Ipanema: a family of cabledriven parallel robots for industrial applications". In CableDriven Parallel Robots. Springer, pp. 119-134.

[3] Lamaury, J., and Gouttefarde, M., 2013. "Control of a large redundantly actuated cable-suspended parallel robot”. In
Robotics and Automation (ICRA), 2013 IEEE International Conference on, IEEE, pp. 4659-4664.

[4] Book, W. J., Hastings, G. G., and Alberts, T. E., 1986. "Design strategies for high-speed lightweight robots".

[5] Rognant, M., Courteille, E., and Maurine, P., 2010. "A systematic procedure for the elastodynamic modeling and identification of robot manipulators". Robotics, IEEE Transactions on, 26(6), pp. 1085-1093.

[6] Pahl, G., and Beitz, W., 2013. Engineering design: a systematic approach. Springer Science \& Business Media.

[7] Angeles, J., and Park, F. C., 2016. "Design and Performance Evaluation”. In Springer Handbook of Robotics. Springer International Publishing, Cham, pp. 399-418.

[8] Khalil, W., and Dombre, E., 2004. Modeling, identification and control of robots. Butterworth-Heinemann.

[9] Siciliano, B., Sciavicco, L., Villani, L., and Oriolo, G., 2010. Robotics: modelling, planning and control. Springer Science \& Business Media.

[10] Singh, T., and Singhose, W., 2002. "Input shaping/time delay control of maneuvering flexible structures". In American Control Conference, 2002. Proceedings of the 2002, Vol. 3, IEEE, pp. 1717-1731.

[11] De Luca, A., and Book, W. J., 2016. "Robots with Flexible Elements". In Springer Handbook of Robotics. Springer International Publishing, Cham, pp. 243-282.

[12] Benosman, M., and Le Vey, G., 2004. "Control of flexible manipulators: A survey”. Robotica, 22(5), pp. 533-545.

[13] Book, W. J., Maizza-Neto, O., and Whitney, D. E., 1975. "Feedback Control of Two Beam, Two Joint Systems With Distributed Flexibility". Journal of Dynamic Systems, Measurement, and Control, 97.

[14] Benner, P., Gugercin, S., and Willcox, K., 2013. “A survey of model reduction methods for parametric systems".

[15] Rout, B., and Mittal, R., 2010. "Optimal design of manipulator parameter using evolutionary optimization techniques". Robotica, 28(03), pp. 381-395.

[16] Rubrecht, S., 2011. "Contributions à la commande de robots sous contraintes". $\mathrm{PhD}$ thesis, Université Pierre et Marie Curie-Paris VI.

[17] Maurice, P., 2015. "Virtual ergonomics for the design of collaborative robots". $\mathrm{PhD}$ thesis, Paris 6.

[18] Totaro, N., and Guyader, J.-L., 2013. "Modal energy analysis". Journal of Sound and Vibration, 332(16), pp. 37353749 . 